九州大学学術情報リポジトリ

Kyushu University Institutional Repository

\title{
Periodic modulation of tubular vesicles induced by phase separation
}

Yanagisawa, Miho

Department of Physics, Graduate School of Science, Kyoto University

Imai, Masayuki

Department of Physics, Ochanomizu University

Taniguchi, Takashi

Department of Chemical Engineering, Graduate School of Engineering, Kyoto University

http://hdl. hand le. net/2324/20284

出版情報: Physical Review E. 82 (5)，pp.051928-1-051928-9，2010-11. American Physical Society バージョン：

権利関係 : 


\title{
Periodic modulation of tubular vesicles induced by phase separation
}

\author{
Miho Yanagisawa, ${ }^{1, *}$ Masayuki Imai, ${ }^{2}$ and Takashi Taniguchi ${ }^{3}$ \\ ${ }^{1}$ Department of Physics, Graduate School of Science, Kyoto University, Sakyo, Kyoto 606-8102, Japan \\ ${ }^{2}$ Department of Physics, Ochanomizu University, Otsuka, Bunkyo, Tokyo 112-8610, Japan \\ ${ }^{3}$ Department of Chemical Engineering, Graduate School of Engineering, Kyoto University, Nishikyo, Kyoto 615-8510, Japan
}

(Received 29 September 2010; published 22 November 2010)

\begin{abstract}
We investigated changes in the shape of tubular ternary vesicles induced by phase separation into liquidordered and liquid-disordered phases. Tubular vesicles transform into two types of periodically modulated vesicles depending on the area fraction of the liquid-ordered phase. One type is a necklace of oblate subunits with two circular domains of the liquid-order phase, and the other is a periodically modulated tube with stripes of the liquid-order phase. The transition between the circular and striped domains is governed by the domain boundary energy, whereas the periodicity of modulated vesicles is determined geometrically based on the fixed volume and area constraints. The observed multidomain vesicles are kinetically trapped in metastable states, and all domains show budding to reduce the boundary energy.
\end{abstract}

DOI: 10.1103/PhysRevE.82.051928

PACS number(s): 87.16.dt, 87.16.dj, 87.14.Cc

\section{INTRODUCTION}

Homogeneous giant unilamellar vesicles (GUVs) show a large variety of shape deformation based on the external conditions, such as a difference in osmotic pressure between the inside and outside of the GUVs [1-3], temperature [4-6], the addition of particular molecules or ions $[7,8]$, and laser irradiation [9]. To understand these observed shape deformations, several theoretical approaches can be applied [10-13]. One of the most successful models among these theoretical approaches is the area difference elasticity (ADE) model [11]. In the ADE model, the total free energy of a vesicle with surface area $S$ and volume $V$ is given by

$$
F=\frac{1}{2} \kappa \int(2 H)^{2} d S+\kappa_{\mathrm{G}} \int K d S+\frac{\alpha \pi \kappa}{2 S d_{\mathrm{m}}^{2}}\left(\Delta S-\Delta S_{0}\right)^{2},
$$

where $d S$ is the area element. The first term in Eq. (1) is the bending energy of the membrane expressed in terms of the bending rigidity $\kappa$ and the mean curvature $H$. The second term also gives bending energy related to the Gaussian curvature $K$ and its modulus $\kappa_{\mathrm{G}}$ and turns out to be constant, since the membrane has homogeneous composition and the topology of the vesicle is fixed. The third term is the ADE energy, which arises from the deviation in the total difference in area between the inner and outer leaflets of the bilayer $\Delta S$ from the intrinsic area difference $\Delta S_{0}$. The intrinsic area difference is defined as $\Delta S_{0}=\left(N_{\text {out }}-N_{\text {in }}\right) a_{0}$, where $a_{0}$ is the cross section of a lipid molecule, and $N_{\text {out }}$ and $N_{\text {in }}$ correspond to the numbers of lipids in the outer and inner leaflets, respectively. This term is expressed using the distance between two monolayers $d_{\mathrm{m}}$ and a numerical constant $\alpha$, which determines the strength of the ADE energy compared to the bending energy. The shape of a homogeneous vesicle is determined by the minimization of $F$ for given $S$ and $V$. Based on this model, the morphology diagram using two parameters, a dimensionless excess area of a vesicle $\xi$, defined as

\footnotetext{
*yanagisawa@chem.scphys.kyoto-u.ac.jp
}

$\xi \equiv(S / 4 \pi)^{1 / 2} /(3 V / 4 \pi)^{1 / 3}-1$, and the intrinsic area difference $\Delta S_{0}$, well describes the observed shape deformations of homogeneous GUVs $[10,14]$.

To obtain a realistic model of biomembranes, we must investigate changes in the shape of GUVs containing various kinds of lipids. In multicomponent GUVs, the interactions between different kinds of lipids lead to the phase separation of lipids [15-19]. For example, a ternary GUV composed of dipalmitoylphosphatidylcholine (DPPC)/ dioleoylphosphatidylcholine (DOPC)/cholesterol (Chol) shows phase separation into a liquid-ordered (Lo) phase that is rich in DPPC and a liquid-disordered (Ld) phase that is rich in DOPC. This phase separation produces heterogeneity of the bending rigidity and line tension between the Lo and Ld phases. In a spherical phase-separated vesicle, the line tension causes domain coarsening to minimize the line energy at domain boundaries. Furthermore, in a nonspherical vesicle with nonzero excess area, the competition between the proportion of bending rigidities in the Lo and Ld phases and the line tension results in unique shape deformations called budding $[18,20]$. This issue has been investigated with both analytical theories [21-27] and computer simulations $[28,29]$. However, to the best of our knowledge, most of these systematic investigations have focused on vesicles with a nearly spherical shape, and the effects of phase separation on nonspherical vesicles with a large excess area are still unclear [30,31]. Baumgart et al. reported a periodically modulated tubular vesicle with a synchronized striped domain pattern, where the membrane was composed of sphingomyelin/DOPC/Chol=0.63:0.07:0.3 [17]. The formation of a multiple domain pattern is very interesting since the domain pattern should be governed by the line energy (typical line tension, $\sigma \cong 10^{-12} \mathrm{~N}$, and typical bending modulus, $\kappa \cong 10^{-19} \mathrm{~J}$ [17], which give a reduced line tension of $\sigma R_{0} / \kappa \cong 50$ for $\left.R_{0}=\sqrt{(S / 4 \pi)}=5 \mu \mathrm{m}\right)$. Recently, we found a similar periodically modulated tubular vesicle composed of $\mathrm{DPPC} / \mathrm{DOPC} / \mathrm{Chol}=4: 4: 2[30,31]$. In our case, upon phase separation, the tubular vesicle transformed to a necklace of oblate subunits with two circular domains of the Lo phase. Thus, phase separation on tubular vesicles causes periodic 
multidomain patterns. The purpose of the present study is to clarify the generality of the periodically modulated tubular vesicles induced by phase separation and to reveal the underlying physics, with particular focus on the circular-to-stripe domain-pattern transition and determination of the wavelength selection of modulation. For this purpose, we present a diagram of the morphology of phase-separated tubular GUVs in the excess area and the area fraction of the Lo phase space. Based on this morphology diagram, we discuss the transition from circular to striped domains and the periodicity of multidomain patterns based on a free-energy analysis and geometrical constraints.

\section{MATERIALS AND METHODS}

\section{A. Materials}

DPPC ( $>99 \%$ purity) and DOPC ( $>99 \%$ purity) were obtained in a powder form from Avanti Polar Lipids (Alabaster, AL). Chol ( $>99 \%$ purity) was purchased from Sigma-Aldrich (St. Louis, MO). All lipids were used without further purification. Mother chloroform solutions of lipids were stored at $-20{ }^{\circ} \mathrm{C}$ until use. Texas-Red dihexanoylphosphatidylethanolamine (TR-DHPE; Molecular Probes, Eugene, OR) was used as a dye for contrast between the Lo and Ld phases. Rhodamine dioleoylphosphatidylethanolamine (Rho-DOPE; Avanti Polar Lipids) and dihexanoylphosphatidylethanolamine- $N$-(7-nitro-2,1,3-benzoxa-diazol-4-yl) (NBD-DHPE; Avanti Polar Lipids) were used for two-color imaging. The former dye partitions into the Ld phase and gives a red color, whereas the latter preferentially partitions into the Lo phase and gives a green color. Sorbitol purchased from Sigma-Aldrich (St. Louis, MO) was used to create a difference in osmotic pressure between the inside and outside of GUVs, which can control the excess area of the vesicles.

\section{B. Preparation of GUVs}

We prepared ternary GUVs composed of DPPC, DOPC, and Chol by the gentle hydration method [18,32]. The area fraction of the Lo phase, $\phi_{0}$, was controlled by varying the mole fraction of Chol, $m_{\text {chol }}=0.15,0.2,0.25,0.3,0.35$, and 0.4 , while keeping the mole ratio of $\mathrm{DPPC} / \mathrm{DOPC}=1: 1$. Roughly speaking, the relationship between $\phi_{\mathrm{o}}$ and $m_{\text {chol }}$ can be expressed as $\phi_{\mathrm{o}} \approx m_{\text {chol }}+0.15\left(0.15<m_{\text {chol }}<0.4\right)$.

First, we dissolved lipids with the prescribed compositions of DPPC, DOPC, and Chol in $10 \mu$ l of chloroform (concentration is $10 \mathrm{mM}$ ). To dye the Ld phase, TR-DHPE was added at $0.2 \mathrm{~mol} \%$ of lipids. For two-color imaging, Rho-DOPE and NBD-DHPE were added at 0.4 and 0.2 mol \% of lipids, respectively. The solvent was evaporated in a stream of nitrogen gas and the obtained lipid film was kept under vacuum overnight to completely remove the remaining solvent. The dried lipid film was prewarmed at $60{ }^{\circ} \mathrm{C}$, and then hydrated with $1 \mathrm{ml}$ of pure water at $60{ }^{\circ} \mathrm{C}$. During hydration process, the lipid films spontaneously form giant vesicles with diameters of 5-50 $\mu \mathrm{m}$.

\section{Fluorescence microscopy observation}

The vesicle suspension was put on a glass plate with a 0.5 -mm-thick silicon rubber spacer. This sample cell was set on a temperature-controlled stage $\left( \pm 0.2^{\circ} \mathrm{C}\right)$ for microscopic observation (Carl Zeiss, Germany). We first kept the temperature of the ternary GUV suspension at $60{ }^{\circ} \mathrm{C}$ in the homogeneous one-phase region and then added sorbitol $(\sim 0.2 \mathrm{mM})$ to create a difference in the osmotic pressure between the inside and outside of GUVs. Upon the addition of sorbitol, spherical homogeneous GUVs started to deform into various morphologies by using the excess area caused by the difference in osmotic pressure. When homogeneous GUVs developed a tubular shape, tubular GUVs of various lengths were quenched to the coexisting two-phase region $\left(24{ }^{\circ} \mathrm{C}\right.$ for $m_{\text {chol }}=0.15,0.2,0.25$, and 0.3 systems and $20^{\circ} \mathrm{C}$ for $m_{\text {chol }}=0.35$ and 0.4 ) [15] at a quenching rate of approximately $30{ }^{\circ} \mathrm{C} / \mathrm{min}$. At this low sorbitol concentration $(\sim 0.2 \mathrm{mM})$, the rate of shape deformation was much slower than that of phase separation, and thus GUVs maintained a constant excess area during phase separation. The intrinsic area difference was also constant due to the slow flip-flop rate of lipids [33]. The process of shape deformation was followed using a confocal fluorescence microscope (LSM5, Carl Zeiss, Germany). For the observation, TR-DHPE and Rho-DHPE were excited with a He-Ne laser $(\lambda=543 \mathrm{~nm})$ and fluorescence was detected through a 560-615 nm bandpass filter (Chroma, Rockingham, VT). NBD-DOPE was excited by an Ar laser $(\lambda=488 \mathrm{~nm})$ and fluorescence was detected through a 505-530 nm bandpass filter (Chroma). To avoid photo-oxidation which can raise the transition temperature and induce phase separation at constant temperature $[15,16]$, we minimized light exposure time and the intensity.

\section{RESULTS AND DISCUSSION}

In this study, we prepared tubular ternary GUVs with various excess areas in a homogeneous one-phase region, and then quenched them into a coexisting two-phase region. With quenching, phase separation into Lo and Ld phases occurred with a change in the shapes of the tubules. We found that a long tubular GUV with a minor Lo phase $\left(\phi_{\mathrm{o}}\right.$ $<0.5)$ transforms to a necklace of oblate subunits with circular Lo domains on the top and bottom as reported in Ref. $[30]$, whereas a GUV with a major Lo phase $\left(\phi_{0}>0.5\right)$ transforms to a periodically modulated tube with stripes of a Lo domain as reported in Ref. [17]. In Fig. 1, we summarize the morphologies of phase-separated vesicles schematically using two key geometrical parameters: $\phi_{\mathrm{o}}\left(m_{\mathrm{chol}}\right)$ and $\xi$. In the vesicle image, dark and bright regions correspond to the Lo and Ld phases, respectively. We estimated $\xi$ of a periodically modulated GUV from the shape of a homogeneous tubular GUV (assuming a spherocylinder shape) just before phase separation. The total surface area and volume of a tubular GUV with length $l$ and radius $r$ are given by $S_{t}=2 \pi r l$ $+4 \pi r^{2}$ and $V_{t}=\pi r^{2} l+4 \pi r^{3} / 3$, respectively, and the value of $\xi$ is obtained by $\xi=\left(S_{t} / 4 \pi\right)^{1 / 2} /\left(3 V_{t} / 4 \pi\right)^{1 / 3}-1$. The observed morphologies were independent of the quenching rate in the range of $30-0.5^{\circ} \mathrm{C} / \mathrm{min}$. This morphology diagram clearly shows the generality of the periodically modulated tubular 


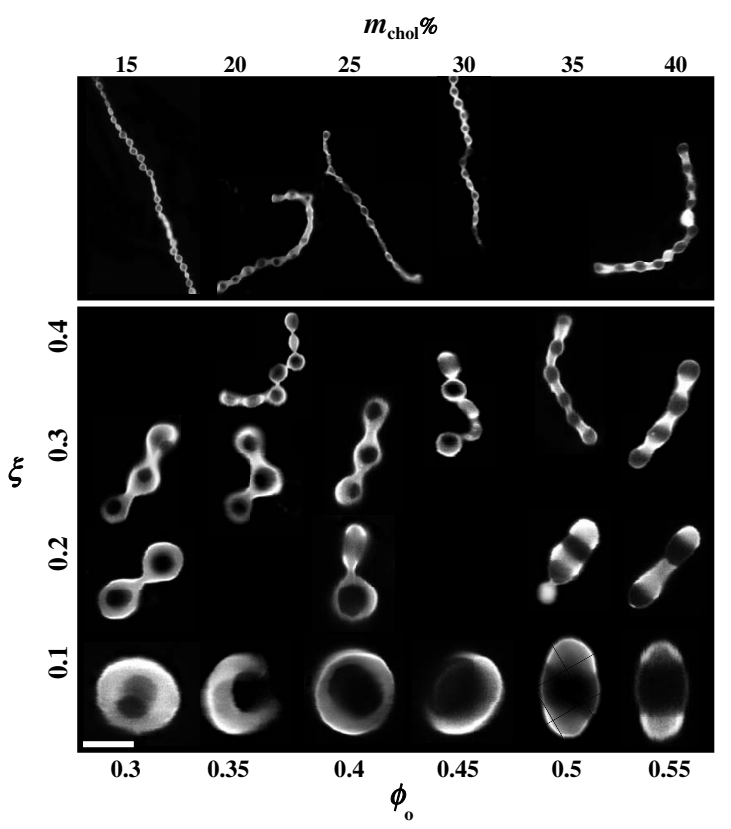

FIG. 1. Morphology diagram of phase-separated tubular vesicles in $\phi_{\mathrm{o}}\left(m_{\text {chol }}\right)$ and $\xi$ space. The vesicles above the thick white line show typical shapes with large $\xi(\sim 0.8)$. The white and black regions in the vesicles are the $\mathrm{Ld}$ and Lo phases, respectively. Scale bar is $5 \mu \mathrm{m}$.

vesicles induced by phase separation. From Fig. 1, we found that (1) with an increase in $\phi_{0}$, the Lo domain pattern changes from the circular to stripe type, accompanied by a change in the vesicle shape from a necklace to a modulated tube, and (2) with an increase in $\xi$, the number of subunits (necklace vesicle) or stripes (modulated tubular vesicle) increases. These two findings are the main points of this paper.

Note that the phase-separated vesicles maintained multidomain patterns for several tens of minutes as metastable states. Then, the domains start to bud and show complete budding within a few minutes after the start of budding. Figure 2 shows some examples of such budding transitions of multidomain vesicles using the two-color imaging technique (green and red regions correspond to the Lo and Ld phases, respectively). For a GUV with $\phi_{0}=0.35$ and $\xi=0.3$ [Fig. 2(a)] and a GUV with $\phi_{0}=0.45$ and $\xi=0.1$ [Fig. 2(b)], phaseseparated vesicles show an oblate-based shape with a circular multidomain pattern. Over time, the circular Lo domains completely budded toward the outside without coarsening, while phase-separated vesicles maintained a multidomain pattern for several tens of minutes. Similarly, for modulated tubular vesicles with a striped multidomain pattern, the domain boundaries between the Lo and Ld phases shrink with time, and the red and green domains are finally connected by a small neck, as shown in Fig. 2(c) $\left(\phi_{0}=0.5\right.$ and $\left.\xi=0.2\right)$ and Fig. 2(d) $\left(\phi_{0}=0.55\right.$ and $\left.\xi=0.6\right)$. These results clearly indicate that the multidomain patterns are metastable, and the line energy governs shape deformation.

In this experiment, phase-separated vesicles were irradiated with possibly low-intensity light at intervals of $>5 \mathrm{~s}$ since continuous exposure to bright light induced budding as an artifact of light. In addition, when we waited for $\sim 1 \mathrm{~h}$ before turning on the light, most of the vesicles already un-

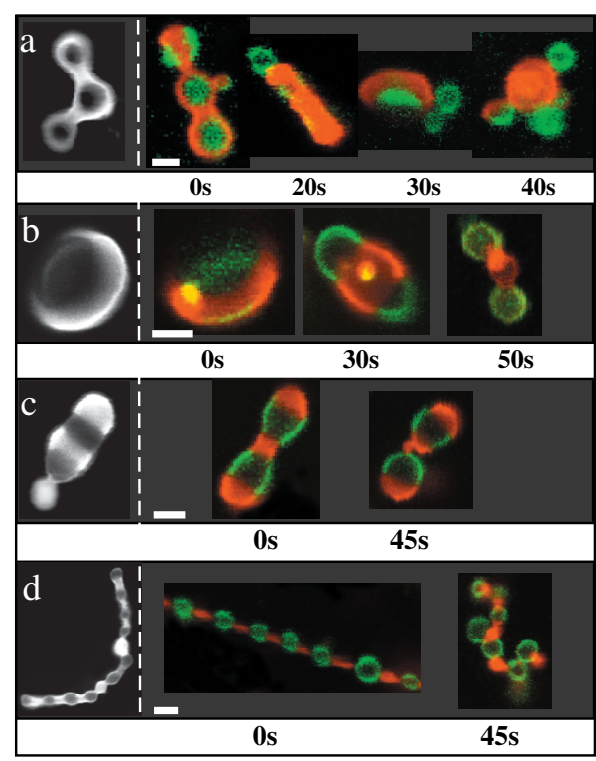

FIG. 2. (Color) Budding transition of multidomains on modulated tubular vesicles with a mole fraction of DPPC/DOPC $=1: 1$ and Chol mole fractions of (a) 0.2, (b) 0.3, (c) 0.35, and (d) 0.4. The values of excess area in each vesicle are (a) 0.3 , (b) 0.1 , (c) 0.2 , and (d) 0.6. The onset time of the budding transition is expressed as $0 \mathrm{~s}$. Red (white) and green (black) regions indicate the Ld and Lo phases, respectively. Scale bars are (a)-(c) 5 and (d) $2 \mu \mathrm{m}$.

derwent budding while some of them did not. Therefore, under our experimental conditions, the effects of light on the metastability of multidomain patterns are not dominant.

\section{A. Domain-pattern transition}

First, we consider the transition from circular to striped domains of the Lo phase in GUVs with small excess area $(\xi<0.1)$, as shown in the bottom row of Fig. 1. In the case of a short tubular vesicle with $\phi_{0}<0.5$, the vesicle transformed to an oblate with two circular domains of the Lo phase on both sides. Since the bending modulus of the Lo phase, $\kappa^{0}$, is greater than that of the Ld phase, $\kappa^{\mathrm{d}},\left(\kappa^{\mathrm{o}} / \kappa^{\mathrm{d}}=1.25-4\right)$ $[17,34]$, the Lo domains prefer less-curved region, whereas the Ld matrix covers the higher-curvature region. With an increase in $\phi_{0}$, the circular domain gradually increased in size while maintaining an oblate shape. When the area fraction of the Lo phase increased beyond a critical value, $\phi_{0}^{*}$ $\sim 0.5$, the tubular vesicle transformed to a prolate vesicle with a single stripe of the Lo phase. A further increase in $\phi_{0}$ resulted in an increase in the thickness of the stripe.

For vesicles with a larger excess area $(\xi>0.1)$, upon phase separation, long tubular vesicles transformed to periodically modulated tubes with a multidomain patterns. Long tubular vesicles with a low $\phi_{0}\left(\phi_{0}<0.5\right)$ showed a necklace composed of oblate subunits with circular Lo domains, whereas for high $\phi_{0}\left(0.5 \leq \phi_{0}<0.65\right)$, vesicles showed modulated tubular shape with synchronized stripes of the Lo phase. Thus, the transition between the necklace with circular domains and the modulated tube with stripes took place at $\phi_{\mathrm{o}}^{*} \sim 0.5$, which is independent of $\xi$. 

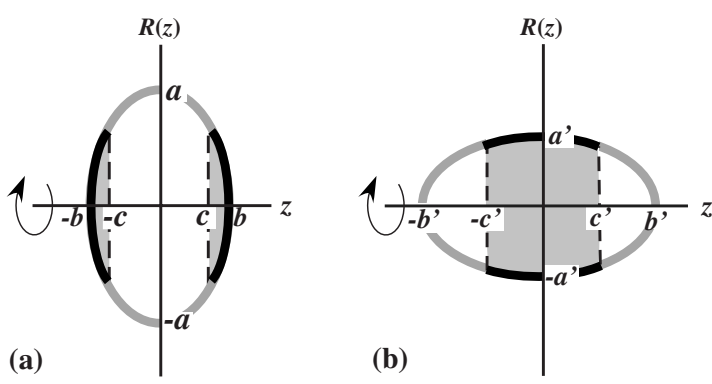

FIG. 3. Geometrical spheroid models describing (a) an axisymmetric oblate vesicle with two circular Lo domains on both sides (OV2CD) and (b) an axisymmetric prolate vesicle with one striped Lo domain (PV1SD). The gray and black lines represent cross sections of the $\mathrm{Ld}$ and Lo phases, respectively.

\section{B. Free-energy analysis of the domain-pattern transition}

Here, we consider the circular-to-stripe domain-pattern transition theoretically. For a short tubular vesicle with $\xi<0.1$ as an initial state, with phase separation, the vesicle deforms to an oblate vesicle with two circular Lo domains (OV2CD) or a prolate vesicle with one striped Lo domain (PV1SD) depending on $\phi_{0}$. Although the observed multidomains in vesicles finally showed budding as seen in Fig. 2, OV2CD and PV1SD maintained their shapes for several tens of minutes before the start of budding. Hence, we addressed the domain-pattern transition based on the total free energy. The elastic energy model for a homogeneous vesicle expressed by Eq. (1) can be extended to a vesicle with multiple domains as follows [30]:

$$
\begin{gathered}
F=F_{\text {bending }}+F_{\text {line }}+F_{\mathrm{ADE}}, \\
F_{\text {bending }}=\sum_{(\mathrm{p})=\mathrm{o}, \mathrm{d}} \int\left\{\frac{1}{2} \kappa^{(\mathrm{p})}(2 H)^{2}+\kappa_{\mathrm{G}}^{(\mathrm{p})} K\right\} d S, \\
F_{\text {line }}=\sum_{i} \sigma \oint d l^{(i)}, \\
F_{\mathrm{ADE}}=\frac{\alpha \pi \bar{\kappa}}{2 S l^{2}}\left(\Delta S-\Delta S_{0}\right)^{2},
\end{gathered}
$$

where the superscript "p" stands for the Lo phase (o) and the Ld phase (d), $\sigma$ is the line tension at the boundary between the Lo and Ld phases, and $d l^{(i)}$ is the length element of the $i$ th domain boundary. The mean bending modulus of the bilayer, $\bar{\kappa}$, is given by $\bar{\kappa}=\kappa^{\mathrm{o}} \phi_{\mathrm{o}}+\kappa^{\mathrm{d}}\left(1-\phi_{\mathrm{o}}\right)$. For this calculation, we approximated OV2CD and PV1SD by an oblate spheroid and a prolate spheroid, as shown in Fig. 3. The details of the analytical expressions of the total volume $V$, total area $S$, area fraction of the Lo phase $\phi_{\mathrm{o}}$, and energy terms for the oblate and prolate vesicles are summarized in Appendix, Secs. 1 and 2, respectively. Although the reported values for line tension have ranged from 0.01 to $5 \mathrm{pN}$ depending on the lipid composition and temperature [17,35-37], under our experimental condition, the total free energy is governed by the line energy, as shown in Fig. 3. Therefore, in the energy calculation, we adopted $\kappa^{\mathrm{O}}=1$
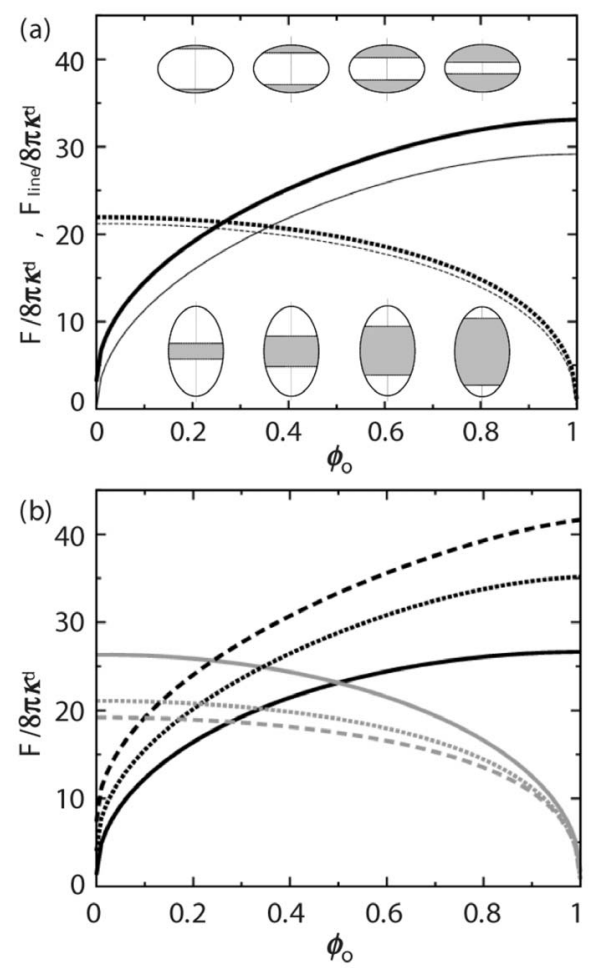

FIG. 4. (a) Normalized total free energy $\left(F / 8 \pi \kappa^{\mathrm{d}}\right.$, thick lines) and normalized line energy $\left(F_{\text {line }} / 8 \pi \kappa^{\mathrm{d}}\right.$, thin lines $)$ curves of OV2CD (solid lines) and PV1SD (dotted lines) with $\xi=0.02$ as functions of the area fraction of the Lo phase, $\phi_{0}$. Schematic representations show the geometries of OV2CD (upper side) and PV1SD (lower side) with different $\phi_{0}=0.2,0.4,0.6$, and 0.8 (left to right). The gray regions represent Lo phases. (b) Normalized total free energy $\left(F / 8 \pi \kappa^{\mathrm{d}}\right)$ for OV2CD (black lines) and PV1SD (gray lines) as a function of $\phi_{0}$. Both vesicles have same excess areas $\xi=0.06$ (dashed lines), 0.03 (dotted lines), and 0 (solid lines).

$\times 10^{-19} \mathrm{~J}, \quad \kappa^{\mathrm{o}} / \kappa^{\mathrm{d}}=1.25, \quad \kappa_{\mathrm{G}}^{\mathrm{o}}=\kappa_{\mathrm{G}}^{\mathrm{d}}$, and $\sigma=1 \mathrm{pN}$, and thus $\sigma R_{0} / \kappa^{\mathrm{d}} \cong 50$ for $R_{0}=5 \mu \mathrm{m}$. We plotted the normalized total free energies $F / 8 \pi \kappa^{\mathrm{d}}$ (thick line) and normalized line energies $F_{\text {line }} / 8 \pi \kappa^{\mathrm{d}}$ (thin line) for OV2CD (solid line) and PV1SD (dotted line) with $\xi=0.02$ against $\phi_{0}$ in Fig. 4(a), which shows a schematic representation of phase-separated vesicles for each $\phi_{0}$ in the upper (OV2CD) and lower (PV1SD) panels. Figure 4(a) shows that the domain boundary length determines the stable shape. With an increase in $\phi_{0}$, the boundary length of a domain on an oblate vesicle $L^{\mathrm{O}}$ increases as $L^{\mathrm{O}} \sim a \phi_{\mathrm{o}}^{1 / 2}$, whereas that on a prolate vesicle $L^{\mathrm{p}}$ decreases as $L^{\mathrm{p}} \sim a^{\prime} \sqrt{1-\phi_{0}^{2}}$. The shape transition from OV2CD to PV1SD takes place at the crossover point shown in Fig. 4(a). The numerically estimated area fraction at the transition is ${ }^{n} \phi_{0}^{*} \sim 0.27$ for vesicles with $\xi=0.02$. This value is smaller than the experimental value, $\phi_{0}^{*} \sim 0.5$. Figure $4(\mathrm{~b})$ shows the normalized free energies of OV2CD and PV1SD with various $\xi$ 's against $\phi_{0}$. The transition area fraction ${ }^{n} \phi_{0}^{*}$ increases with a decrease in $\xi$. When the vesicle has a spherical shape with $\xi=0$, the theoretical crossover area fraction is ${ }^{n} \phi_{\mathrm{o}}^{*}=0.5$, which agrees well with the experimental value.

Similar domain-pattern transitions between circular and striped domains have been observed in membranes supported 


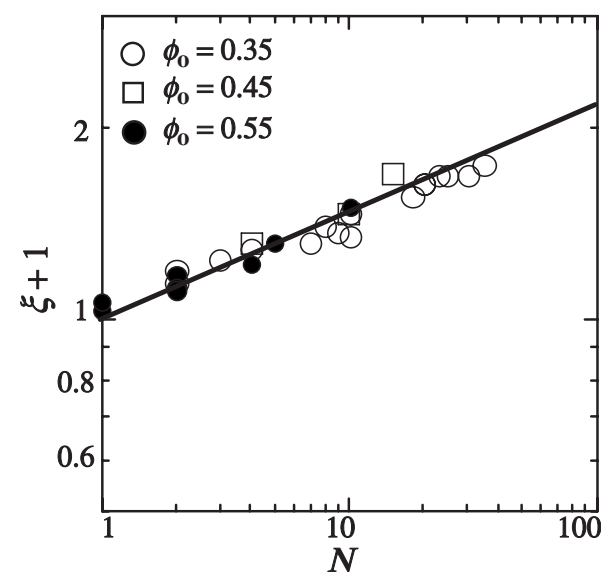

FIG. 5. Relationship between the number of subunits $N$ and excess area $\xi$ for periodically modulated vesicles with various $\phi_{0}$ 's. Necklace vesicles with $\phi_{0}=0.35$ and $\phi_{0}=0.45$ are composed of $N$ oblate subunits, and tubular vesicles with $\phi_{0}=0.55$ have $N$ striped Lo domains. The solid line indicates the geometrical relationship $\xi+1=\left(\xi_{0}+1\right) N^{1 / 6}$, with $\xi_{0}=0$.

on substrates [38-41]. Rozycki et al. showed that circular domains represent the most stable state if the line tension is sufficiently high, whereas striped domain patterns correspond to the equilibrium state if the line tension is lower than a threshold value [39]. Domain-pattern transitions between circular and striped domains controlled by line tension have also been reported for vesicles using a curvature elasticity model $[21,24,27]$. In our experimental condition, however, the line energy is large enough to dominate the total energy $\left(\sigma R_{0} / \kappa_{\mathrm{d}} \gg 1\right)$. Actually, all domains in circular and striped patterns showed a budding transition (Fig. 2). Therefore, the observed domain patterns are metastable states, and the domain-pattern transition occurs so as to minimize the line energy.

\section{Periodicity of modulated vesicles}

The second feature of shape deformation in phaseseparated tubular vesicles is the periodic modulation shown in Fig. 1. With phase separation, a homogeneous tubular vesicle with small $\xi(<0.1)$ deforms to OV2CD $\left(0.35<\phi_{\text {o }}\right.$ $<0.50)$ or PV1SD $\left(0.50 \leq \phi_{0}<0.65\right)$. When the value of $\xi$ increases beyond $\sim 0.1$, the shape of the phase-separated vesicle is transformed to a dimer of oblate subunits $(0.35$ $\left.<\phi_{\mathrm{o}}<0.50\right)$ or a modulated tube with two Lo stripes $(0.50$ $\left.\leq \phi_{\mathrm{o}}<0.65\right)$. The number of subunits in a periodic vesicle, $N$, increases with an increase in $\xi$. In the case of long tubular vesicles with $\xi \sim 0.8, N$ reaches approximately 40 .

Figure 5 shows the experimentally obtained relationship between $\xi$ and $N$ on a double-logarithmic plot. For $\phi_{0}$ $=0.35$ and 0.45 , the vesicles have a necklace shape, and for $\phi_{\mathrm{o}}=0.55$ the vesicles have a modulated tubular shape. All of the data obtained for necklaces and modulated tubes follow a master curve of $(\xi+1) \sim N^{v}$, with $\nu=0.16$. This result indicates that one subunit corresponds to one wave of modulation in the tube.

To explain this geometrical relationship, we introduce a dimensionless excess area of a subunit defined by

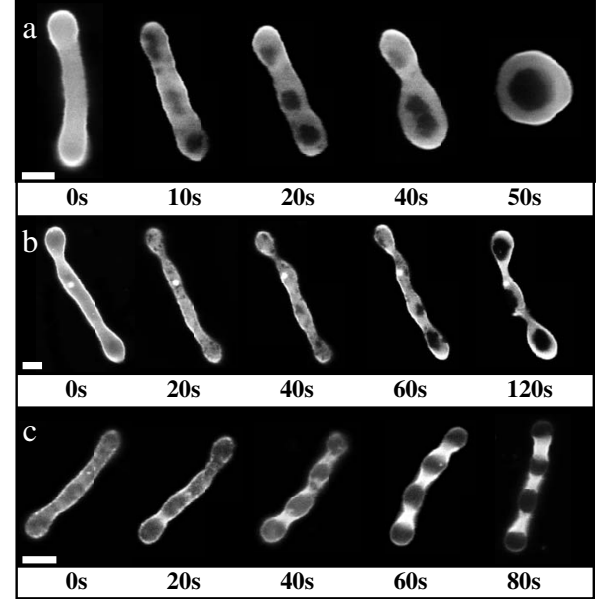

FIG. 6. Time evolution of domain growth on tubular vesicles with (a) $\phi_{0}=0.35, \xi=0.1$; (b) $\phi_{0}=0.35, \xi=0.3$; and (c) $\phi_{0}=0.55$, $\xi=0.3$. The onset time of visible phase separation is expressed as $0 \mathrm{~s}$. The white and black regions are the Ld and Lo phases, respectively. The scale bar is $5 \mu \mathrm{m}$.

$\xi_{0}=\left(s_{0} / 4 \pi\right)^{1 / 2} /\left(3 v_{0} / 4 \pi\right)^{1 / 3}-1$, where $s_{0}$ and $v_{\mathrm{o}}$ are the area and volume of the subunit, respectively. Since the total area and volume of the periodic vesicle are given by $S_{p}=N s_{0}$ and $V_{p}=N v_{0}$, we obtain the following expression:

$$
\xi+1=\frac{\left(S_{p} / 4 \pi\right)^{1 / 2}}{\left(3 V_{p} / 4 \pi\right)^{1 / 3}}=\left(\xi_{0}+1\right) N^{1 / 6} .
$$

Equation (6) well describes the experimental data in Fig. 5. From fitting, we obtain $\xi_{0} \sim 0$. This value agrees well with the estimated value $\xi$ in the analysis using free energy. Thus, the periodicity of a modulated vesicle, i.e., the number of subunits $N$, is determined by the geometrical conservation constraints of $S$ and $V$, and each subunit is nearly spherical.

\section{Kinetic pathways to periodically modulated vesicles}

To reveal the relationship between multidomain-pattern formation and periodic deformation in the vesicle, the time evolutions of phase-separated tubular vesicles are shown in Fig. 6. In the case of a tubular vesicle with $\xi=0.1$ and $\phi_{0}$ $=0.35[$ Fig. $6(\mathrm{a})]$, the coarsening of numerous small domains occurred in three regions (10 s), which developed to threedomain pairs (in the front and back sides) on the modulated tubular vesicle $(20 \mathrm{~s})$. While the three-domain-pair state did not develop to a trimer vesicle, the domains coalesced to one-domain pair in an oblate vesicle, OV2CD (50 s). Similar coarsening of the domain pattern and simultaneous shape deformation were observed in other tubular vesicles. In the case of a tubular vesicle with $\xi=0.3$ and $\phi_{0}=0.35$ [Fig. 6(b)], the coarsening of domains took place in four regions (40 s), and the vesicle formed a four-domain-pair state on the modulated tubular vesicle $(60 \mathrm{~s})$. However, the domains finally developed to three-domain pairs on a trimer vesicle (120 s). Thus, the periodicity of the initial domain pattern does not always agree with that of the modulated vesicle. On the other hand, for a tubular vesicle with $\xi=0.3$ and $\phi_{0}=0.55$ [Fig. $6(c)]$, the number of stripes on a vesicle was independent of 


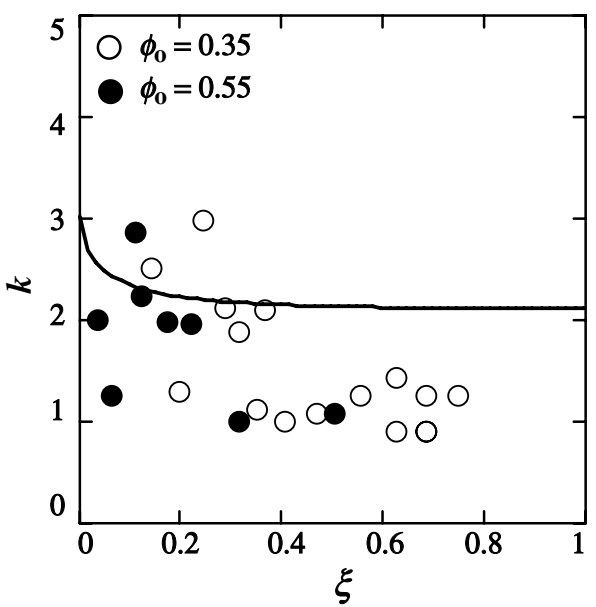

FIG. 7. Dimensionless wave number $k=2 \pi r / \lambda$ of modulated vesicles $\left(\phi_{0}=0.35\right.$ : necklace of oblate subunits and $\phi_{0}=0.55$ : tubular vesicles with striped domains) as a function of the excess area $\xi$. The solid line indicates the geometrical relationship $\xi=-1+\left(\xi_{0}\right.$ $+1)\left\{(k / \pi)^{2}(3-2 \pi / k)^{-1}\right\}^{1 / 6}$, with $\xi_{0}=0$.

time, which may be due to the high-energy barrier for the coalescence of stripe domains.

\section{E. Mechanism of periodic modulation induced by phase separation}

The formation of periodically modulated tubular vesicles with multiple domains is quite interesting. Campelo et al. addressed modulated vesicles with striped domains by energy minimization in the Canhan-Helfrich model [42]. However, the line tension $\left(\sigma R_{0} / \kappa_{\mathrm{d}}<1\right)$ adopted in their paper is much smaller than that in our system $\left(\sigma R_{0} / \kappa_{\mathrm{d}} \sim 50\right)$. Therefore, it might be worthwhile to discuss other possibilities to explain the formation of periodically modulated vesicles. The periodic instability in homogeneous tubular vesicles has been observed in many systems and is referred to as pearling instability [9,43-45]. For pearling instability, two types of theoretical models have been presented: a hydrodynamic theory for a fluid membrane based on plateau and Rayleigh instability $[46,47]$ and a membrane elasticity model with nonzero spontaneous curvature [48]. According to the former theory, pearling instability originates from an excess surface tension induced by an external stimulus and has a wavelength of the fastest growth mode, $\lambda$, as $2 \pi r / \lambda \approx 0.65$, where $r$ is the radius of the tube [45]. In the latter theory, pearling is a result of the minimization of the membrane elastic energy with nonzero spontaneous curvature, which leads to strings of beads, the so-called unduloid or "Delaunay surfaces" with $2 \pi r / \lambda=1$, where $\lambda$ is the wavelength of modulation [48-50]. To compare these theoretical predictions with our experimental data, we plot the dimensionless wave number $k$ $=2 \pi r / \lambda$ of the periodically modulated vesicles as a function of $\xi$ (Fig. 7). For large $\xi(>0.4), k$ is about 1 , which means that the vesicle shape is close to "unduloid." On the other hand, for small $\xi(<0.4), k$ is scattered at around 2 . When a tubular vesicle with length $l$ and radius $r$ transforms into a necklace composed of $N$ spheres with radius $R$, the total surface area and total volume are

$$
\begin{aligned}
& S=2 \pi r l+4 \pi r^{2}=4 \pi R^{2} N, \\
& V=\pi r^{2} l+\frac{4}{3} \pi r^{3}=\frac{4}{3} \pi R^{3} N,
\end{aligned}
$$

respectively. Equations (6)-(8) and $\lambda=2 R$ give the following geometrical relation:

$$
\xi=-1+\left(\xi_{0}+1\right)\left\{\left(\frac{k}{\pi}\right)^{2}\left(3-2 \frac{\pi}{k}\right)^{-1}\right\}^{1 / 6} .
$$

We plotted Eq. (9) with $\xi_{0}=0$ as a solid line in Fig. 7. The wave number $k$ calculated by Eq. (9) agrees with the experimental results for small $\xi<0.4$ region. Thus, Fig. 7 shows that the shapes of short and long tubular vesicles can be described by the necklace and unduloid geometries, respectively. To reveal the physical basis of the observed periodically modulated vesicles, further studies are needed.

\section{CONCLUSION}

When we induce phase separation in tubular vesicles, the tubular vesicles transform into two types of periodically modulated vesicles depending on the area fraction of the Lo phase. One type is a necklace of oblate subunits with two circular Lo domains, and the other is a modulated tubular vesicle with stripes of the Lo phase. The circular-to-stripe domain transition is governed by the line energy, whereas the periodicity of modulated vesicles is determined geometrically by volume and the area conservation constraints. For a vesicle with small $\xi<0.4$, the shape is a necklace of subunits with the same $\xi \sim 0$. However, for large $\xi>0.4$, the shape is close to an unduloid. Since the observed multidomains are maintained for several minutes and then show a budding transition with time, they are metastable states. Furthermore, the shape deformation of vesicles upon phase separation is governed by a kinetic process, which is completely different from the shape deformation of homogeneous vesicles and may be important for understanding the more complex morphologies of biomembranes.

\section{ACKNOWLEDGMENTS}

This work was supported by a Grant-in-Aid for Scientific Research on Priority Area "Soft Matter Physics" from the Ministry of Education, Culture, Sports, Science and Technology of Japan and by a Grant-in-Aid for Scientific Research (B) No. 19340118 from the Japan Society for the Promotion of Science. M.Y. is grateful for support from the Japan Society for the Promotion of Science (JSPS Grant-in-Aid No. 22-940).

\section{APPENDIX: ESTIMATION OF FREE ENERGY OF OV2CD AND PV1SD}

In this appendix, we derive the free-energy expression of the two types of phase-separated vesicles OV2CD and PV1SD. The shapes of OV2CD and PV1SD are approximated by an oblate spheroid [Fig. 3(a)] and a prolate spheroid [Fig. 3(b)] with axisymmetry with respect to the $z$ axis, 
respectively. The radius $R(z)$ of the spheroids in the cylindrical coordinate system is expressed as

$$
R(z)=a \sqrt{1-\left(\frac{z}{b}\right)^{2}}
$$

The surfaces of the spheroids are divided into the Lo and Ld phases, as shown in Fig. 3 [(a) oblate and (b) prolate spheroids].

\section{Oblate shape}

The OV2CD has radii of major, $a$, and minor, $b$, axes, i.e., $a \geq b$ and the phase boundaries are located at $z= \pm c$ $(c \leq b)$. The total volume $V$ and surface area $S$ of the oblate vesicle are given by

$$
V=\frac{4}{3} \pi a^{2} b
$$

$$
S=2 \pi\left\{a^{2}+\frac{a b^{2}}{2 \sqrt{a^{2}-b^{2}}} \ln \left|\frac{a+\sqrt{a^{2}-b^{2}}}{a-\sqrt{a^{2}-b^{2}}}\right|\right\} .
$$

The areas of the Lo phase $S_{\mathrm{o}}$ and Ld phase $S_{\mathrm{d}}$ are expressed as

$$
S_{\mathrm{o}}=S-S_{\mathrm{d}}
$$

$$
\begin{aligned}
S_{\mathrm{d}}= & 2 \pi\left[\frac{a c}{b^{2}} \sqrt{b^{4}+\left(a^{2}-b^{2}\right) c^{2}}\right. \\
& \left.+\frac{a b^{2}}{2 \sqrt{a^{2}-b^{2}}} \ln \left|\frac{\sqrt{b^{4}+\left(a^{2}-b^{2}\right) c^{2}}+c \sqrt{a^{2}-b^{2}}}{\sqrt{b^{4}+\left(a^{2}-b^{2}\right) c^{2}}-c \sqrt{a^{2}-b^{2}}}\right|\right],
\end{aligned}
$$

and the area fraction of Lo phase, $\phi_{0}$, is given by

$$
\phi_{\mathrm{o}}=\frac{S_{\mathrm{O}}}{S} \text {. }
$$

The bending energy is expressed as

$$
\begin{aligned}
F_{\text {bending }}= & 2 \pi\left(\frac{a}{b}\right) \frac{1}{p}\left(\kappa ^ { \mathrm { 0 } } \left\{\frac{1}{2} \ln \left|\frac{a+\sqrt{a^{2}-b^{2}}}{a-\sqrt{a^{2}-b^{2}}} \frac{\sqrt{b^{2}+p^{2} c^{2}}+p c}{\sqrt{b^{2}+p^{2} c^{2}}-p c}\right|+2\left(\frac{a^{2}}{b^{2}}\right)\left(\frac{\sqrt{a^{2}-b^{2}}}{a}-\frac{p c}{\sqrt{b^{2}+p^{2} c^{2}}}\right)+\frac{1}{3}\left(\frac{a^{2}}{b^{2}}\right)^{2}\left[\frac{\left(2 a^{2}+b^{2}\right) \sqrt{a^{2}-b^{2}}}{a^{3}}\right.\right.\right. \\
& \left.\left.-\frac{p c\left(3 b^{2}+2 p^{2} c^{2}\right)}{\left(b^{2}+p^{2} c^{2}\right)^{3 / 2}}\right]\right\}+\kappa^{\mathrm{d}}\left[\frac{1}{2} \ln \left|\frac{a+\sqrt{a^{2}-b^{2}}}{a-\sqrt{a^{2}-b^{2}}}\right|+2\left(\frac{a^{2}}{b^{2}}\right) \frac{\sqrt{a^{2}-b^{2}}}{a}+\frac{1}{3}\left(\frac{a^{2}}{b^{2}}\right)^{2} \frac{\left(2 a^{2}+b^{2}\right) \sqrt{a^{2}-b^{2}}}{a^{3}}\right] \\
& \left.+2 \kappa_{\mathrm{G}}^{\mathrm{o}}\left(\frac{\sqrt{a^{2}-b^{2}}}{a}-\frac{p c}{\sqrt{b^{2}+p^{2} c^{2}}}\right)+2 \kappa_{\mathrm{G}}^{\mathrm{d}} \frac{p c}{\sqrt{b^{2}+p^{2} c^{2}}}\right),
\end{aligned}
$$

where $p \equiv\left(a^{2} / b^{2}-1\right)^{1 / 2}$. The line and ADE energies are

$$
\begin{gathered}
F_{\text {line }}=4 \pi \sigma \frac{a}{b}\left(b^{2}-c^{2}\right)^{1 / 2} \\
F_{\mathrm{ADE}}=8 \pi^{3} \alpha \bar{\kappa} \frac{1}{S}\left[\frac{a^{2}}{\sqrt{a^{2}-b^{2}}} \arctan \left(\frac{\sqrt{a^{2}-b^{2}}}{b}\right)+b\right. \\
\left.-\frac{1}{4 \pi d_{\mathrm{m}}} \Delta S_{0}\right]^{2}
\end{gathered}
$$

\section{Prolate shape}

The PV1SD is also expressed as a spheroid with the radii of minor, $a^{\prime}$, and major, $b^{\prime}$, axes, i.e., $a^{\prime} \leq b^{\prime}$ and the width of a stripe domain, $2 c^{\prime}\left(c^{\prime} \leq b^{\prime}\right)$ as shown in Fig. 3(b). The total volume $V$ and surface area $S$ of the prolate spheroid are, respectively, given by

$$
\begin{gathered}
V=\frac{4}{3} \pi a^{\prime 2} b^{\prime}, \\
S=2 \pi\left\{a^{\prime 2}+\frac{a^{\prime} b^{\prime 2}}{\sqrt{b^{\prime 2}-a^{\prime 2}}} \arccos \left(\frac{a^{\prime}}{b^{\prime}}\right)\right\} .
\end{gathered}
$$

The area of the Lo phase, $S_{0}$, is expressed as

$$
\begin{aligned}
S_{\mathrm{o}}= & 2 \pi\left\{\frac{a^{\prime} c^{\prime}}{b^{\prime 2}} \sqrt{b^{\prime 4}-\left(b^{\prime 2}-a^{\prime 2}\right) c^{\prime 2}}\right. \\
& \left.+\frac{a^{\prime} b^{\prime 2}}{\sqrt{b^{\prime 2}-a^{\prime 2}}} \arccos \left(\frac{\sqrt{b^{\prime 4}-\left(b^{\prime 2}-a^{\prime 2}\right) c^{\prime 2}}}{b^{\prime 2}}\right)\right\},
\end{aligned}
$$

and the area of the Ld phase, $S_{\mathrm{d}}$, is given by $S_{\mathrm{d}}=S-S_{\mathrm{o}}$. The area fraction of the Lo phase, $\phi_{0}$, is given by $\phi_{0}=S_{0} / S$. The bending energy is expressed as 


$$
\begin{aligned}
F_{\text {bending }}= & 2 \pi\left(\frac{a^{\prime}}{b^{\prime}}\right) \frac{1}{q}\left(\kappa^{\mathrm{o}}\left\{\arcsin \left(q \frac{c^{\prime}}{b^{\prime}}\right)+2\left(\frac{a^{\prime 2}}{b^{\prime 2}}\right) \frac{\sqrt{\left(b^{\prime 2}-a^{\prime 2}\right) c^{\prime 2}}}{\sqrt{b^{\prime 4}-\left(b^{\prime 2}-a^{\prime 2}\right) c^{\prime 2}}}+\frac{1}{3}\left(\frac{a^{\prime 2}}{b^{\prime 2}}\right)^{2} \frac{\left(3 b^{\prime 2}-2 c^{\prime 2} q^{2}\right) b^{\prime 4} c^{\prime} q}{\left[b^{\prime 4}-\left(b^{\prime 2}-a^{\prime 2}\right) c^{\prime 2}\right]^{3 / 2}}\right\}\right. \\
& +\kappa^{\mathrm{d}}\left(\arcsin (q)-\arcsin \left(q \frac{c^{\prime}}{b^{\prime}}\right)+2\left(\frac{a^{\prime 2}}{b^{\prime 2}}\right)\left[\frac{\sqrt{b^{\prime 2}-a^{\prime 2}}}{a^{\prime}}-\frac{\sqrt{\left(b^{\prime 2}-a^{\prime 2}\right) c^{\prime 2}}}{\sqrt{b^{\prime 4}-\left(b^{\prime 2}-a^{\prime 2}\right) c^{\prime 2}}}\right]+\frac{1}{3}\left(\frac{a^{\prime 2}}{b^{\prime 2}}\right)^{2}\left\{\frac{\left(3-2 q^{2}\right) b^{\prime 3} q}{a^{\prime 3}}\right.\right. \\
& \left.\left.\left.-\frac{\left(3 b^{\prime 2}-2 c^{\prime 2} q^{2}\right) b^{\prime 4} c^{\prime} q}{\left[b^{\prime 4}-\left(b^{\prime 2}-a^{\prime 2}\right) c^{\prime 2}\right]^{3 / 2}}\right\}\right)+2 \kappa_{\mathrm{G}}^{\mathrm{o}} \frac{\sqrt{\left(b^{\prime 2}-a^{\prime 2}\right) c^{\prime 2}}}{\sqrt{b^{\prime 4}-\left(b^{\prime 2}-a^{\prime 2}\right) c^{\prime 2}}}+2 \kappa_{\mathrm{G}}^{\mathrm{B}}\left[\frac{\sqrt{b^{\prime 2}-a^{\prime 2}}}{a^{\prime}}-\frac{\sqrt{\left(b^{\prime 2}-a^{\prime 2}\right) c^{\prime 2}}}{\sqrt{b^{\prime 4}-\left(b^{\prime 2}-a^{\prime 2}\right) c^{\prime 2}}}\right]\right),
\end{aligned}
$$

where $q \equiv\left(1-a^{\prime 2} / b^{\prime 2}\right)^{1 / 2}$. The line energy is given by

$$
F_{\text {line }}=4 \pi \sigma \frac{a^{\prime}}{b^{\prime}}\left(b^{\prime 2}-c^{\prime 2}\right)^{1 / 2} .
$$

The ADE energy is

$$
F_{\mathrm{ADE}}=8 \pi^{3} \alpha \bar{\kappa} \frac{1}{S}\left[\frac{1}{2} \frac{a^{\prime 2}}{\sqrt{b^{\prime 2}-a^{\prime 2}}} \ln \left(\frac{\sqrt{b^{\prime 2}-a^{\prime 2}}+b^{\prime}}{\sqrt{b^{\prime 2}-a^{\prime 2}}-b^{\prime}}\right)+b^{\prime}-\frac{1}{4 \pi l} \Delta S_{0}\right]^{2}
$$

[1] E. Boroske, M. Elwenspoek, and W. Helfrich, Biophys. J. 34, 95 (1981).

[2] H. Hotani, J. Mol. Biol. 178, 113 (1984).

[3] A. L. Bernard, M. A. Guedeau-Boudeville, L. Jullien, and J. M. di Meglio, Biochim. Biophys. Acta 1567, 1 (2002).

[4] K. Berndl, J. Kas, R. Lipowsky, E. Sackmann, and U. Seifert, EPL 13, 659 (1990).

[5] J. Kas and E. Sackmann, Biophys. J. 60, 825 (1991).

[6] H. G. Dobereiner, J. Kas, D. Noppl, I. Sprenger, and E. Sackmann, Biophys. J. 65, 1396 (1993).

[7] B. L. Mui, H. G. Dobereiner, T. D. Madden, and P. R. Cullis, Biophys. J. 69, 930 (1995).

[8] R. Sano, S. M. Masum, T. Tanaka, Y. Yamashita, V. Levadny, and M. Yamazaki, J. Phys.: Condens. Matter 17, S2979 (2005).

[9] R. Bar-Ziv, E. Moses, and P. Nelson, Biophys. J. 75, 294 (1998).

[10] U. Seifert, Adv. Phys. 46, 13 (1997).

[11] S. Svetina and B. Zeks, Eur. Biophys. J. 17, 101 (1989).

[12] U. Seifert, K. Berndl, and R. Lipowsky, Phys. Rev. A 44, 1182 (1991).

[13] H. W. Gerald-Lim, M. Wortis, and R. Mukhopadhyay, in Lipid Bilayers and Red Blood Cells, Soft Matter Vol. 4, edited by G. Gompper and M. Schick (Wiley-VCH, New York, 2008), p. 83.

[14] P. Ziherl and S. Svetina, EPL 70, 690 (2005).

[15] S. L. Veatch and S. L. Keller, Phys. Rev. Lett. 89, 268101 (2002).

[16] S. L. Veatch and S. L. Keller, Biochim. Biophys. Acta 1746, 172 (2005).

[17] T. Baumgart, S. T. Hess, and W. W. Webb, Nature (London) 425, 821 (2003).

[18] M. Yanagisawa, M. Imai, T. Masui, S. Komura, and T. Ohta, Biophys. J. 92, 115 (2007).

[19] Y. Sakuma, M. Imai, M. Yanagisawa, and S. Komura, Eur. Phys. J. E 25, 403 (2008).
[20] R. Lipowsky and R. Dimova, J. Phys.: Condens. Matter 15, S31 (2003).

[21] F. Jülicher and R. Lipowsky, Phys. Rev. Lett. 70, 2964 (1993).

[22] T. Taniguchi, K. Kawasaki, D. Andelman, and T. Kawakatsu, J. Phys. II 4, 1333 (1994).

[23] T. Taniguchi, Phys. Rev. Lett. 76, 4444 (1996).

[24] F. Jülicher and R. Lipowsky, Phys. Rev. E 53, 2670 (1996).

[25] W. T. Góźdź and G. Gompper, Phys. Rev. E 59, 4305 (1999).

[26] W. T. Góźdź, Langmuir 24, 12458 (2008).

[27] E. Gutlederer, T. Gruhn, and R. Lipowsky, Soft Matter 5, 3303 (2009).

[28] P. B. Sunil Kumar, G. Gompper, and R. Lipowsky, Phys. Rev. Lett. 86, 3911 (2001).

[29] M. Laradji and P. B. Sunil Kumar, Phys. Rev. Lett. 93, 198105 (2004).

[30] M. Yanagisawa, M. Imai, and T. Taniguchi, Phys. Rev. Lett. 100, 148102 (2008).

[31] M. Yanagisawa, M. Imai, and T. Taniguchi, Prog. Theor. Phys. Suppl. 175, 71 (2008).

[32] J. P. Reeves and R. M. Dowben, J. Cell Physiol. 73, 49 (1969).

[33] J. Liu and J. C. Conboy, Biophys. J. 89, 2522 (2005).

[34] S. Semrau, T. Idema, L. Holtzer, T. Schmidt, and C. Storm, Phys. Rev. Lett. 100, 088101 (2008).

[35] C. Esposito, A. Tian, S. Melamed, C. Johnson, S. Y. Tee, and T. Baumgart, Biophys. J. 93, 3169 (2007).

[36] A. Tian, C. Johnson, W. Wang, and T. Baumgart, Phys. Rev. Lett. 98, 208102 (2007).

[37] A. R. Honerkamp-Smith, P. Cicuta, M. D. Collins, S. L. Veatch, M. den Nijs, M. Schick, and S. L. Keller, Biophys. J. 95, 236 (2008).

[38] R. Parthasarathy, C. H. Yu, and J. T. Groves, Langmuir 22, 5095 (2006).

[39] B. Różycki, T. R. Weikl, and R. Lipowsky, Phys. Rev. Lett. 100, 098103 (2008).

[40] S. Rozovsky, Y. Kaizuka, and J. T. Groves, J. Am. Chem. Soc. 127, 36 (2005). 
[41] S. Komura, N. Shimokawa, and D. Andelman, Langmuir 22, 6771 (2006).

[42] F. Campelo, J. M. Allain, and M. Ben Amar, EPL 77, 38006 (2007).

[43] S. Chaïeb and S. Rica, Phys. Rev. E 58, 7733 (1998).

[44] I. Tsafrir, D. Sagi, T. Arzi, M. A. Guedeau-Boudeville, V. Frette, D. Kandel, and J. Stavans, Phys. Rev. Lett. 86, 1138 (2001).

[45] T. T. Nguyen, A. Gopal, K. Y. C. Lee, and T. A. Witten, Phys.
Rev. E 72, 051930 (2005).

[46] P. Nelson, T. Powers, and U. Seifert, Phys. Rev. Lett. 74, 3384 (1995).

[47] L. Rayleigh, Philos. Mag. 34, 145 (1892).

[48] F. Campelo and A. Hernandez-Machado, Phys. Rev. Lett. 99, 088101 (2007).

[49] H. J. Deuling and W. Helfrich, Blood Cells 3, 713 (1977).

[50] Ou-Yang Zhong-can and W. Helfrich, Phys. Rev. A 39, 5280 (1989). 